\title{
BMJ Open Study protocol for a randomised controlled trial evaluating the effectiveness of strengths model case management (SMCM) with Chinese mental health service users in Hong Kong
}

Samson Tse, ${ }^{1}$ Sau Man Catalina Ng, ${ }^{2}$ Wing Yan Winnie Yuen, ${ }^{1}$ Sadaaki Fukui, ${ }^{3}$ Richard J Goscha, ${ }^{4}$ Wann Ka Iris Lo ${ }^{1}$

To cite: Tse S, Ng SMC, Yuen WYW, et al. Study protocol for a randomised controlled trial evaluating the effectiveness of strengths model case management (SMCM) with Chinese mental health service users in Hong Kong. BMJ Open 2019;9:e026399. doi:10.1136/ bmjopen-2018-026399

- Prepublication history and additional material for this paper are available online. To view these files, please visit the journal online (http://dx.doi. org/10.1136/bmjopen-2018026399).

Received 5 September 2018 Revised 19 March 2019 Accepted 18 April 2019
Check for updates

(C) Author(s) (or their employer(s)) 2019. Re-use permitted under CC BY-NC. No commercial re-use. See rights and permissions. Published by BMJ.

For numbered affiliations see end of article.

Correspondence to Professor Samson Tse; samsont@hku.hk

\section{ABSTRACT}

Introduction Strengths-based approaches mobilise individual and environmental resources that can facilitate the recovery of people with mental illness. Strengths model case management (SMCM), developed by Rapp and Goscha through collaborative efforts at the University of Kansas, offers a structured and innovative intervention. As evidence of the effectiveness of strengths-based interventions come from Western studies, which lacked rigorous research design or failed to assure fidelity to the model, we aim to fill these gaps and conduct a randomised controlled trial (RCT) to test the effectiveness of SMCM for individuals with mental illness in Hong Kong.

Methods and analysis This will be an RCT of SMCM. Assuming a medium intervention effect (Cohen's $\mathrm{d}=0.60$ ) with $30 \%$ missing data (including dropouts), 210 service users aged 18 years or above will be recruited from three community mental health centres. They will be randomly assigned to SMCM groups (intervention) or SMILE groups (control) in a 1:1 ratio. The SMCM groups will receive strengths model interventions from case workers, whereas the SMILE groups will receive generic care from case workers with an attention placebo. The case workers will all be embedded in the community centres and will be required to provide a session with service users in both groups at least once every fortnight. There will be two groups of case workers for the intervention and control groups, respectively. The effectiveness of the SMCM will be compared between the two groups of service users with outcomes at baseline, 6 and 12 months after recruitment. Functional outcomes will also be reported by case workers. Data on working alliances and goal attainment will be collected from individual case workers. Qualitative evaluation will be conducted to identify the therapeutic ingredients and conditions leading to positive outcomes. Trained outcome assessors will be blind to the group allocation.

Ethics and dissemination Ethical approval from the Human Research Ethics Committee at the University of Hong Kong has been obtained (HRECNCF: EA1703078). The results will be disseminated to service users and their
Strengths and limitations of this study

- First study using a randomised controlled trial and a rigorous mixed-methods design to assess the effectiveness of strengths model case management (SMCM) conducted in Hong Kong and worldwide. Fidelity or integrity of the SMCM intervention will be monitored to explore its effects on service users' outcomes.

- Evidence of implementing SMCM among Chinese in community mental health settings will be established and the key therapeutic ingredients of SMCM will be identified.

- This study provides a unique opportunity to examine how the SMCM should be adapted to ensure practice and services are culturally responsive (based on our best knowledge about SMCM, we have made preliminary cultural adaptations (eg, translation of the assessment, explaining the concept of strength) before the trial).

- Case workers of SMCM and SMILE (control) groups in the same community mental health centre may share the same philosophy (eg, recovery approach) and culture of the agency leading to potential contamination between the two groups.

- Service users may not stay in the community centre (due to withdrawing from the study or being discharged from the service) for the entire 12-month trial period.

families via the media, to healthcare professionals via professional training and meetings and to researchers via conferences and publications.

Trial registration number 12617001435370; Pre-results.

\section{INTRODUCTION}

Common mental disorders (CMD) such as depression and anxiety are very prevalent in the population globally. It is estimated that 
over 300 million people experience depression worldwide. ${ }^{1}$ A population-based study, Hong Kong Mental Morbidity Survey 2010-2013, reported that $13.3 \%$ and $2.5 \%$ of adults aged 16-75 years were diagnosed with $\mathrm{CMD}^{2}$ and psychotic disorders, ${ }^{3}$ respectively. The consequences of CMD are reflected in the results of the 2010 Global Burden of Diseases, Injuries and Risk Factors Study (GBD 2010), ${ }^{4}$ which reported that bipolar disorder and schizophrenia accounted for $7.0 \%$ and $7.4 \%$ of disability-adjusted life years, respectively. In addition, the global costs of mental illness were estimated at US\$2.5 trillion in 2010 and this figure is set to more than double to US $\$ 6$ trillion by 2030.5 Mental illness not only affects the physical and psychological health of the individuals concerned but also their families, caregivers and friends. Therefore, better treatment and planning of care are essential to promote recovery from mental illness and improve outcomes.

Across the globe, traditional treatments for mental illness primarily consist of medication. ${ }^{6}$ However, mental illness is often long-lasting and recurrent, so taking medication alone may not be sufficient to achieve personal recovery, which is broadly defined as 'a deeply personal, unique process of changing one's attitudes, values, feelings, goals, skills and/or roles ... a way of living a satisfying, hopeful and contributing life even with the limitations caused by illness'. ${ }^{7}$ It also refers to the process of individual psychological adaptation to an illness and addressing functional impairment. ${ }^{8}$ With the proliferation of positive psychology advocated by Seligman, ${ }^{9}$ more empirical studies focus on strengths of character, which are defined as 'positive traits reflected in thoughts, feelings, and behaviors' (Park, p603) ${ }^{10}$ and positive experiences such as life satisfaction. ${ }^{10}$ In recent years, more researchers have advocated strengths-based approaches, which mobilise individual and environmental resources that can facilitate recovery among people with mental illness. ${ }^{11}{ }^{12}$ Strengths model case management (SMCM) has gained increasing favour among practitioners since the 1980s in Kansas. ${ }^{1314}$

\section{Conceptual framework: Kansas model, SMCM}

SMCM was developed by Rapp and Goscha through collaborative efforts at the University of Kansas (KU).1213 The Kansas model of SMCM aims to enhance recovery through instilling hope and empowering the choices and autonomy of service users, rather than focusing on deficits. Case management refers to the process of assessing needs, implementing a service plan and monitoring progress to bring about positive outcomes. ${ }^{1516}$

SMCM consists of four main core elements, including strengths assessment, personal recovery plan, group supervision and field mentoring. The strengths assessment is used to appraise the service users' strengths, niches in the community and other attributes such as hope, self-efficacy and the resources available in the family and community, while the personal recovery plan uses the information obtained from the strengths assessment to derive a plan that consists of recovery goals that are meaningful to the service users. Coconstruction of recovery goals between service users and case workers can be challenging, ${ }^{17}$ so the steps towards achieving a goal should be small, specific and measurable. Group supervision aims to provide a supportive environment for case workers to help service users achieve the goals they identify. Furthermore, field mentoring will be provided to case workers on a regular basis or whenever necessary. It has been designed to help staff develop and refine their SMCM practice skills in the field with service users.

SMCM is guided by six major principles: (1) people with mental illness have the capacity to recover, reclaim and transform their lives; (2) the focus is on the strengths of individuals; (3) the community is perceived as an oasis of resources; (4) the service user is the director of the helping process; (5) the worker-service user relationship is essential; and (6) the primary setting of the work is the community. ${ }^{13}$ In addition, SMCM lays emphasis on three themes. First, case workers should be creative about how to help each service user achieve a life that brings purpose, meaning and a valued identity. Second, SMCM does not neglect the problems and impediments that service users are facing. Instead, problems and obstacles are perceived within the context of the goals that the service user desires to achieve. Third, SMCM is not only about a change in clinical practice but also requires the transformation of our care systems such as policies and the way we communicate with each other to best support service users in finding niches within their community in which they can thrive. Figure 1 shows the important concepts of the SMCM framework.

Most studies evaluating the effectiveness of SMCM in helping people with mental illness reported positive outcomes in many areas such as rehospitalisation, housing, employment, social support, family burden and symptoms. ${ }^{11}{ }^{18-25}$ Similarly, in a recent review, ${ }^{26}$ the results showed that the benefits associated with the strengthsbased approach included reductions in the duration of stay

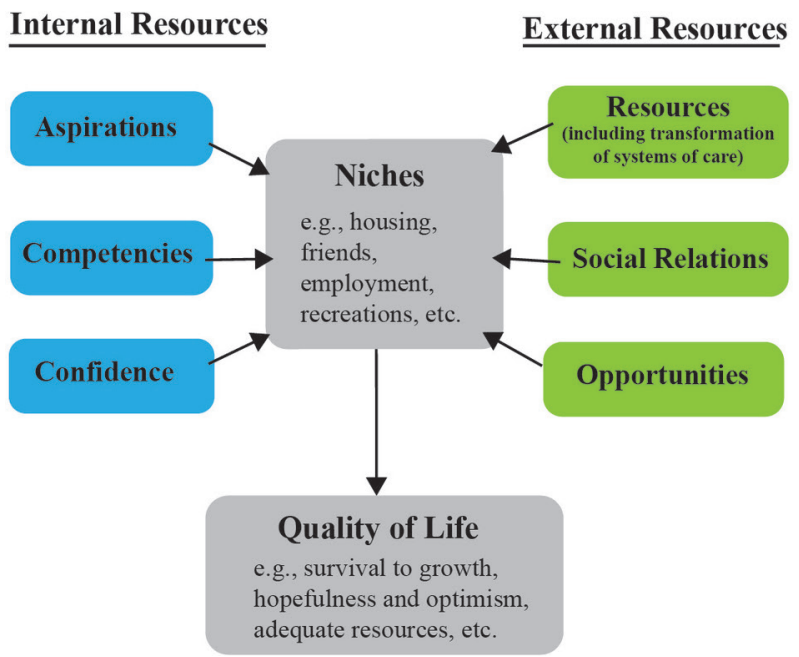

Figure 1 Salient concepts of strengths model case management (modified from Rapp and Goscha, p50)[13]. 
in hospital, increases in service satisfaction, improvements in general attitudes towards recovery-relevant dimensions (eg, self-efficacy and sense of hope), improvements in employment and educational outcomes, increases in the utilisation of general services and increases in job satisfaction as well as morale among mental health professionals. SMCM offers a promising alternative to traditional approaches focusing on service users' deficits.

Nevertheless, as the majority of studies about SMCM have been conducted in the Western context, its use and outcomes lack cultural sensitivity. Based on our best knowledge about SMCM, we have made preliminary cultural adaptations (eg, translating the forms used in the strengths assessment and personal recovery plan, using local terms and examples to explain the concept of strength) before the trial. The current study will provide the best opportunity to investigate SMCM's cultural (eg, under the influence of Taoism, Chinese people tend to be more reserved in stating their strengths and successes) and structural (eg, the caseload sizes, the ratio of supervisor to case worker and so on) compatibility and to understand how SMCM can be best implemented in community mental health settings such as Integrated Community Centres for Mental Wellness (ICCMWs). According to the model ${ }^{12}$ the structure of the service, including a low caseload size, a low supervisor to case worker ratio and the presence of structured supervision sessions, are vital to the implementation and effectiveness of SMCM. However, the structure of mental health services is different in Hong Kong compared with the USA, for example, the caseload in mental health services remains high (the Hospital Authority in Hong Kong reported that in 2016, ratio of case worker to individual with severe mental illness residing in the community was around 1:47 on average), therefore it might be a factor affecting the results of SMCM. Only intervention teams who have achieved high-fidelity scores will be included in the present trial (for details, see the Method section).

Furthermore, reviews of the existing literature indicate that there are limitations to the existing studies. First, among 11 empirical studies ${ }^{180-22} 25$ 27-32 that have investigated the effectiveness of SMCM in mental healthcare settings, only a single study used a randomised controlled trial (RCT) design, which is considered to be the gold standard ${ }^{33}$ in research, and other studies used either secondary data analyses, quasi-experimental with a predesign and postdesign, or between-group comparison. Second, the RCTs on so-called 'strengths-based interventions' were all conducted prior to the year 2000. Furthermore, none of the studies used fidelity assessment to ensure that the intervention group was actually using the strengths model. ${ }^{26}$ Thus, the proposed study seeks to fill this gap in the existing body of literature. Next, results from recent studies showed that SMCM fidelity affects intervention outcomes across service sites, with high-fidelity SMCM associated with lower rates of psychiatric hospitalisation and higher employment rates. ${ }^{32}$ However, little is known about the important constituent of the strengths model approach and how the fidelity scores (or distribution across different items) have impacted on service users' recovery outcome. Finally, the conceptualisation of strengths is culturally defined through linguistics, metaphors, icons and folklore traditions. ${ }^{34}$ Chinese people view their strengths as ever-changing, universal and dialectical in nature, as well as being shaped by their upbringing/family traditions and the lived experience of mental illness. Introspection is critical in the discovery of strengths, which could be influenced by the Taoist philosophy and the Confucius' the Doctrine of Mean (Zhongyong 中庸). ${ }^{34} 35$ Therefore, preliminary cultural adaptation of SMCM before implementing it in a Chinese (or, more broadly, Asian) context is of paramount importance. ${ }^{35}$

\section{Study objectives}

Building on the experience of conducting an earlier trial using a non-randomised design ${ }^{36}$ in a residential setting, the primary objective of the present study is to assess the effectiveness of SMCM when implemented among service users in ICCMWs in Hong Kong, using an RCT design. Specifically, we hypothesise that service users in the high-fidelity SMCM group will experience higher levels of personal recovery, as well as symptom reductions, improved hope, community integration, mattering, working alliances and goal attainment, relative to their counterparts in a control group, which incorporates an attention placebo. The second objective is to evaluate the fidelity features of SMCM implementation and the major therapeutic ingredients that have an effect on service users' outcomes.

\section{METHODS}

\section{Study design and setting}

By choosing a mixed-methods design, we will use quantitative methods to evaluate the effectiveness of SMCM on multidimensional outcomes, while a qualitative study will be used to examine the fidelity features (eg, structure and supervision) and therapeutic ingredients that may be related to the intervention outcomes. These two methods supplement each other. In terms of the settings, the study will take the form of an RCT to examine the effectiveness of SMCM for service users with mental illness. It will consist of two arms: an SMCM intervention group and a control group (called 'SMILE' group-not an acronym). The service users in both arms will be recruited from the ICCMWs of three non-governmental organisations (NGOs). To achieve diversity in the study samples, the NGOs joining the present study are located in different districts in Hong Kong. Service users attending ICCMWs are individuals with either suspected mental health problems or diagnosed mental illnesses.

\section{Sample size and statistical power}

We performed Monte Carlo simulations to estimate the required sample size by using the methods recommended 
by Muthén and Muthén. ${ }^{37}$ We assumed a medium effect size (Cohen's $d=0.6$ ) for the slope (change rate) difference of the primary outcome measure (a mean slope difference between the intervention and control groups) based on previous empirical studies. We assumed 15\% and $30 \%$ missing data at 6 and 12 months, respectively. ${ }^{26} \mathrm{~A}$ total of 210 service users ( $\mathrm{n}=105$ per group) will be required for a statistical power of 0.80 to detect a medium effect with the amount of missing data taken into consideration. We adopted the fixed-effects model in accounting for the cluster effects given the small cluster size (eg, three centres).

\section{Participants}

We will recruit a total of 210 service users from three ICCMWs. On the arrival of the service users, a trained social worker will undertake the recruitment. He/she will be responsible for screening the eligibility of service users based on the inclusion and exclusion criteria. The inclusion criteria include as follows.

i. Being a service user of mental health services in ICCMWs.

ii. Being aged 18 years or above.

iii. Being Chinese; the ability to read Chinese and speak Cantonese.

iv. Being diagnosed with mental illness, including major depressive disorder, anxiety disorder, bipolar disorder and psychotic disorders, by a psychiatrist.

v. The ability to provide written informed consent to join the study and a willingness to be allocated to either group (SMCM or SMILE).

The exclusion criteria will be applied to those service users who are as follows.

i. Likely to engage in immediate risk behaviour, such as suicide and/or violence.

ii. Affected by overt psychotic symptoms and unable to sustain a meaningful conversation for $>10 \mathrm{~min}$ as identified by case workers.

Incentives in the form of supermarket coupons (worth HK\$50) will be given to the service users at all three time points, as compensation for participating in the study.

\section{Recruitment of case workers}

The case workers responsible for the delivery of SMCM intervention are ICCMWs staff (eg, registered social worker, programme worker, occupational therapist, nurse). They must have received prior training on SMCM provided by $\mathrm{KU}$ and agree to participate in ongoing group supervision (see the Intervention groups: SMCM group section). There is a standard, 2-day training on SMCM covering nine aspects of the practices such as day 1 on 'Recovery-Illuminating the Path of Hope', 'Strengths Assessment-Amplifying Wellness' and day 2 on 'Relationship/Engagement-Partners in Recovery' and 'Resource Acquisition-Community Mental Health'. The programme details are included as online supplementary information.

\section{Randomisation}

After screening for their eligibility, all service users will be asked to complete a face-to-face baseline questionnaire $\left(\mathrm{T}_{0}\right)$. The social worker will contact the research team to obtain group allocation information and participants will be randomly allocated to either the SMCM or SMILE group in a 1:1 ratio, according to a predetermined randomisation list generated by an online randomisation programme (http://www.randomization.com). Block randomisation will be performed to reduce bias and achieve balance in allocating participants into the intervention and control groups. Rolling enrolment will be used.

Participants with the group assignment will then be followed up by case workers using the SMCM or SMILE protocol and will be asked to complete the same questionnaires at 6 months $\left(\mathrm{T}_{1}\right)$ and 12 months $\left(\mathrm{T}_{2}\right)$. All case workers are required to conduct a session with service users in both intervention and control groups at least once every fortnight. Service user participants will be informed that there are two forms of psychosocial intervention, but they do not know which intervention they are allocated to. They can withdraw from the study at any time. The case workers in either the intervention or control group will not be blind to the group allocation as they will be the ones who provide the services to the users, but the trained outcome assessors who are peer researchers, will be blind to such information. In order to minimise contamination, case workers in the SMILE group will not be provided with any of the tools related to SMCM (eg, strengths assessment, personal recovery plan) or receive any strengths-based supervision. Figure 2 shows the Consolidated Standards of Reporting Trials diagram,

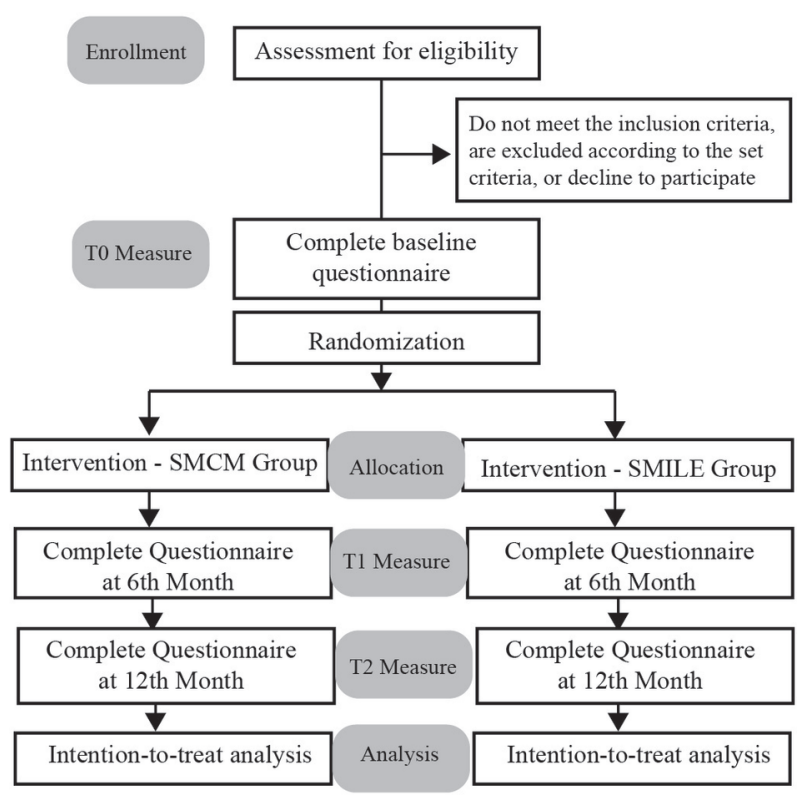

Figure 2 CONSORT diagram reflecting the flow of participants through the current study. CONSORT, Consolidated Standards of Reporting Trials; SMCM, strengths model case management. 
which illustrates the flow of participants throughout the research process.

\section{Intervention groups: SMCM group}

The service user participants allocated to the SMCM group will receive individual sessions of about $30 \mathrm{~min}$ every fortnight. The SMCM intervention can only be delivered by case workers who have previously received training provided by KU. Case workers and supervisors will attend ongoing monthly group supervision led by Goscha (SMCM's author) via Skype. The strengths assessment and personal recovery plan developed by the KU team will be used to guide the intervention sessions. During the intervention, the case workers will help the service users identify recovery goals that are meaningful to them and workers will attend weekly strengths-based supervision run at the ICCMWs. The Fidelity Scale of the service unit will be used every 6 months to monitor if these high-fidelity activities take place as expected. A leadership team consisting of a unit-in-charge and a SMCM supervisor will be established in each ICCMW to oversee the activities.

\section{Control groups: SMILE group}

Service users in the SMILE control group will receive a generic intervention (ie, treatment as usual) which will include recovery groups, medical appointments, leisure/ hobby groups and general community activities. Case workers will have fortnightly contact with service users, which will serve as the attention placebo. Case workers in the control group will call service users on the phone or will meet with them in person for some groups and centre activities. Table 1 summarises the key characteristics of the SMCM intervention and SMILE control groups.

To ensure the integrity of the intervention used in the SMCM and SMILE groups, as well as the adherence to the model, two raters with a thorough understanding of SMCM will closely monitor the intervention by using the Fidelity Scale at 1 (baseline), 6 and 12 months after the intervention starts. The Fidelity Scale was developed to ensure that all components adhered to the model when SMCM was implemented. The scale is composed of nine items across three areas: structure, supervision/ supervisor and clinical/service. In order to qualify to participate in the present study, SMCM teams will need to achieve an overall fidelity score of 36 (out of 45) on the Fidelity Scale including an average rating of 4 out of 5 in each of the three core areas. The fidelity data will be collected through interviews with staff and service users, site observations and reviews of the SMCM tools and charts. The fidelity scores can be improved by three main methods: (1) providing the team with specific comments about which item $(\mathrm{s})$ are under-scored (ie, scores $<4),(2)$

\begin{tabular}{lll}
\hline Table 1 Key characteristics of SMCM intervention and SMILE control groups \\
\hline Dimensions & SMILE group & SMCM group \\
\hline $\begin{array}{ll}\text { 1. Intervention integrity } \\
\text { and infrastructure }\end{array}$ & $\begin{array}{l}\text { No routine fidelity review for the } \\
\text { implementation of recovery-oriented } \\
\text { services. }\end{array}$ & $\begin{array}{l}\text { To ensure a supportive strengths model context through } \\
\text { the Fidelity Scale, which was designed to assess the } \\
\text { adequacy of SMCM implementation in three core areas: } \\
\text { structure, supervision/supervisor and clinical/service. }\end{array}$
\end{tabular}

\section{Individual sessions}

$\begin{array}{ll}\begin{array}{l}\text { a. Strengths } \\ \text { assessments }\end{array} & \begin{array}{l}\text { There is no specific tool for } \\ \text { assessing personal strengths, } \\ \text { therefore they would not be } \\ \text { assessed by the case worker in a } \\ \text { structured way. }\end{array} \\ \text { b. Recovery plans } & \text { Work on specific goals. }\end{array}$

3. Group supervision Adopt the existing supervision arrangements. 
having deeper discussions with the team about the difficulties they are facing and (3) learning from group supervision and field mentoring.

\section{Patient and public involvement}

Given the increasing importance of the role played by patients and public involvement in research and the study objectives, which aim to examine the effectiveness of SMCM, we involved the service users when we conducted a pilot study in September 2017. Three service users from each ICCMW (ie, a total of nine service users) provided their comments on the design of our questionnaire, which helped further refine the wording and clarity of the questionnaire. Second, we will recruit people with lived experience of mental illness as the paid field workers to collect data during the trial. They will undergo training in the university, will be shown how to work with the research participants (eg, responding to commonly asked questions) and will receive ongoing support and coaching. Third, the final results of the study will be disseminated to study participants and the wider public through public fora and seminars organised by the partnered agencies and the funder.

\section{Outcome measures}

The Recovery Assessment Scale (RAS) ${ }^{38}$ will be used to evaluate the primary outcomes, that is, to say, personal recovery in five areas, including personal confidence and hope, willingness to ask for help, goal and success orientation, reliance on others and no domination by symptoms. ${ }^{1831}$ The items will be rated on a 5-point Likert scale ranging from 1 (strongly disagree) to 5 (strongly agree), with a higher score indicating greater perceived recovery. The scale has been used in an RCT of a peer-led program $^{39}$ and in a cross-cultural study. ${ }^{1}$ RAS has the benefit that it has already gone through the cross-cultural adaptation process; it has been translated into Chinese, ${ }^{39}$ yielding good internal consistency and concurrent and construct validity. Other secondary outcomes include validated scales on state of hope, level of symptoms, community integration, mattering, working alliance, as well as goal achievements, functional outcomes and sociodemographic information will also be collected from service user participants. Additionally, working alliance and goal achievements of service users will be collected from case workers. The details of the measurements for measuring the primary and secondary outcomes are summarised in table 2.

\section{Process evaluation}

We will conduct a qualitative study parallel to the RCT to address the study objectives concerning the therapeutic ingredients of SMCM and the link between fidelity features and service users' recovery outcomes. The therapeutic ingredients of SMCM that will be identified are individual, such as state of hope, and environmental (such as community integration) strengths and resources, which help people with mental illness live a meaningful life and integrate into society. We will invite a total of 21 service users and 9 case workers from the intervention group and control group, respectively, for individual interviews at the end of the 12-month assessment period $\left(\mathrm{T}_{2}\right)$. Therefore, seven service users and three case workers will be chosen from each of the three intervention and control sites using a maximum variation technique, which is a form of purposive sampling. ${ }^{40}$ The selection of extreme and typical participants will be based on the richness of strengths identified in the strengths assessment at $T_{2}$ and the manner of completion (eg, using the service users' language and explaining the significance of the strengths to the person). Service user participants who do not use the strengths assessment intensely will also be involved in the qualitative study. A semistructured interview protocol will be developed and used during the interviews to explore the perceived therapeutic ingredients, perceptions of the benefits, challenges in the care and support taking place in either intervention or control group, and suggestions for improvement among the service user and case worker participants. These interviews will be conducted at the ICCMWs for the sake of convenience.

\section{Statistical analyses}

The quantitative data collected will be entered by the research assistants and at least $10 \%$ of the data will be checked. The data cleaning process, including checking the range and missing items, will be performed by research assistants under the supervision of the research team. We will examine the effects of the SMCM intervention on participant outcomes compared with the SMILE group. Background information, including sociodemographic characteristics and all outcome variables, will be summarised using means and SD for continuous variables and frequencies, percentages and cross-tabulations for categorical variables. Univariate and multivariate outliers, histograms, probability plots and residual plots will be examined to select the best-fitting models. We will then conduct growth curve modeling ${ }^{41}$ to test whether or not there are any postintervention significant improvements in participants' outcomes (ie, RAS, hope, community integration, symptoms levels) across three time points at baseline, 6 months and 12 months. Service users will be nested within case workers who are nested within agencies (implementation sites). Given the small sample size at the worker and agency levels, a fixed-effects model will be used at the agency level to control for potential agency effects. Model fit will be evaluated using the multiindex approach, ${ }^{42}$ based on the root mean square error of approximation (RMSEA values $<0.08$ are acceptable, but RMSEA values $<0.05$ are preferred) and comparative fit index (CFI values $>0.90$ are acceptable, but CFI values $>0.95$ are preferred). Full information maximum likelihood will be used to estimate the model, which is also an appropriate method for handling missing data. ${ }^{43}$

Regarding the qualitative data, we will use grounded theory methodology to guide the analysis. ${ }^{44} 45$ Grounded theory provides a systematic framework around which 
Table 2 Measurements used for measuring primary and secondary outcomes

\begin{tabular}{|c|c|c|c|}
\hline $\begin{array}{l}\text { Outcome } \\
\text { measure }\end{array}$ & Measurement & Details of the measurement & Completed by \\
\hline \multicolumn{4}{|c|}{ Primary outcome } \\
\hline $\begin{array}{l}\text { Personal } \\
\text { recovery }\end{array}$ & $\begin{array}{l}\text { Recovery } \\
\text { Assessment Scale }\end{array}$ & $\begin{array}{l}\text { Evaluates the primary outcome of personal recovery in five areas, } \\
\text { including personal confidence and hope, willingness to ask for } \\
\text { help, goal and success orientation, reliance on others and no } \\
\text { domination by symptoms. } 4849 \\
24 \text { items; 5-point Likert scale ranging from 'strongly disagree' } \\
\text { (1) to 'strongly agree' (5), with a higher score indicating greater } \\
\text { perceived recovery. }\end{array}$ & Service users \\
\hline
\end{tabular}

Secondary outcomes

State of hope State Hope Scale ${ }^{50}$ Measures an individual's feelings of hope concerning ongoing Service users events.

- 6 items, 8-point Likert scale ranging from 'definitely false' (1) to 'definitely true' (8).

\begin{tabular}{|c|c|c|c|}
\hline Mattering & Mattering Scale ${ }^{51}$ & $\begin{array}{l}\text { Measures service user participants' perception of the degree to } \\
\text { which they matter to their friends and family. } \\
3 \text { items from the 'Reliance' subscale; 5-point Likert scale ranging } \\
\text { from 'strongly disagree' (1) to 'strongly agree' (5). }\end{array}$ & Service users \\
\hline $\begin{array}{l}\text { Goal } \\
\text { achievement }\end{array}$ & $\begin{array}{l}\text { Created by research } \\
\text { team }\end{array}$ & $\begin{array}{l}\text { A series of questions to gauge participants' goals and progress of } \\
\text { goal achievement. } \\
\text { Service user participants will report goals in areas such as } \\
\text { employment, housing and study or leisure and rate how } \\
\text { meaningful to them, ranging from 'not meaningful at all' (1) to very } \\
\text { meaning (5), and the progress of achieving these goals, ranging } \\
\text { from 'very unsatisfactory' (1) to 'very satisfactory' (5). }\end{array}$ & $\begin{array}{l}\text { Service users } \\
\text { and case } \\
\text { workers }\end{array}$ \\
\hline \multirow[t]{2}{*}{$\begin{array}{l}\text { Working } \\
\text { alliance }\end{array}$} & $\begin{array}{l}\text { Working Alliance } \\
\text { Inventory- } \\
\text { client }^{55} 56\end{array}$ & $\begin{array}{l}\text { Evaluates how well the relationship between the service user and } \\
\text { case worker. } \\
12 \text { items; 7-point Likert scale ranging from 'never' (1) to 'always' } \\
\text { (7). }\end{array}$ & Service users \\
\hline & $\begin{array}{l}\text { Working Alliance } \\
\text { Inventory- } \\
\text { therapist }^{5557}\end{array}$ & $\begin{array}{l}\text { Evaluates how well the relationship between the service user and } \\
\text { case worker. } \\
12 \text { items; 7-point Likert scale ranging from 'never' (1) to 'always' } \\
\text { (7). }\end{array}$ & Case workers \\
\hline
\end{tabular}

interview data can be analysed and interpreted through the use of coding techniques and the constant comparative method. The entire interview process will be audio recorded and transcribed, subsequent to analysis. Quality checks of the transcripts will be performed by a research assistant, who will compare recordings to transcripts for any missing or unclear words. Each interview will be coded in NVivo. The data will be subject to four layers of analysis. ${ }^{46}$ The first layer of analysis will involve organising the material by employing the General Inductive ethod. ${ }^{47}$ The researchers will read the transcripts multiple times, develop a coding framework and further read and discuss based on this framework. The second layer of analysis will involve conceptualising key content into broad themes according to their relevance to the research objective, pertaining to the relationships among the fidelity features of SMCM implementation, therapeutic ingredients and intervention outcomes. The third 
layer will involve further discussion and reading; a limited number of key narratives will identify phases, pathways, processes and mechanisms that operate in relation to SMCM implementation and intervention outcomes. The final layer will explore the extent of convergence and divergence between these different narratives. Convergent themes help identify the common pathways that are likely to be involved in explaining SMCM intervention outcomes. Divergent themes help identify alternatives that may or may not involve increases in positive responses to SMCM intervention. The research rigour of the results will be strengthened by the following two methods. First, the triangulation of data sources refers to carefully reading and comparing the fidelity assessment data and interview findings obtained from service users and case workers. Second, we will perform member checking by sending a summary of the findings to all the participants joining the interviews for comments and check whether the researchers' interpretations of the data match the participants' perceptions of their experiences.

\section{DISCUSSION}

The search for effective interventions to promote recovery from mental illness and improve outcomes for service users should be a priority for mental health services. Although SMCM has been widely implemented in the Western context, its application and effectiveness in non-Western contexts have not been well-studied. The current study aims to fill in the gaps.

Successful confirmation of the effectiveness of SMCM can have theoretical, clinical and societal contributions. First, the findings will generate new knowledge about SMCM that will significantly extend and refine the existing literature on strengths-based practice in mental health such as which section(s) of the Fidelity Scale may account for the service users' improved outcomes. Second, mental health workers often believe that they are already providing recovery-oriented care. Nevertheless, they do not have specific tools to guide their practices. So if proved to be effective, SMCM can provide robust, structured, evidence-based guidelines for strengths-based practices in Hong Kong and other countries. In addition, more service users with mental illness will benefit from the interventions. Lastly, by identifying service users' strengths and showing them how to achieve their aspirations, a strengths-based approach can help minimise the stigmatisation of service users who are often negatively perceived by the rest of the population. This may in turn help people with mental illness integrate into the community and improve their subjective well-being.

Further studies should also consider a longer follow-up period to assess the sustainability of the intervention effects. Furthermore, more studies on the cost-effectiveness of SMCM could be carried out to provide new information pertaining to economic evaluations of SMCM for health policymakers.

\section{ETHICS AND DISSEMINATION}

Written consent will be obtained from all participants and they will be provided with a detailed explanation of the study objectives, the voluntary nature of their participation, their right to withdraw and the risks and benefits of the study. The SMCM is an intervention which should not cause any physical or psychological harm to the participants. However, in case of any unanticipated problem arising or if the participant experiences any discomfort or distress when filling out the questionnaire, or answering the questions in the interview, the field worker should report this to the data-collection supervisor (WKIL and ST). The researchers (ST and WYWY) will help the participants to seek additional support from professionals. Also, the participants may choose not to answer the questions or to terminate the interview. The participants will be asked to sign two copies of the consent letter, with one copy given to the participants and the other one returned to the principal investigator of the present study for record keeping purposes. The consent forms will be kept separately from the data. All data collected, without personal identifiers, will be stored in the principal investigator's (PI) locked filing cabinet, whereas all digital or electronic recordings will be password protected and kept in the PI's computer for 5 years. Only the PI (ST) and the local coinvestigators (SMCN and WYWY) of this research project will have access to the original trial dataset and solely for research purposes.

The current RCT and process evaluation will improve our understanding of the impact of SMCM on service users' recovery, specifically, it will:

1. Demonstrate the benefits and unintended consequences of recovery-oriented, strengths-based services for individuals with mental illness.;

2. Highlight the key therapeutic ingredients of SMCM and how they affect SMCM outcomes.

3. Examine how best to implement SMCM in a Chinese community.

Our approach to knowledge translation will target several key audiences. To disseminate our findings to service users and their families, the PI and the team will work with the local community and media. Healthcare professionals will benefit from the study's contribution to workforce training and professional meetings. We will disseminate our findings to researchers both locally and internationally through conference presentations and publications in peer-reviewed journals. Our results will also be disseminated through seminars organised by the PI's department and the websites of the participating NGOs.

\section{Author affiliations}

${ }^{1}$ Department of Social Work and Social Administration, The University of Hong Kong, Hong Kong, HKSAR, China

${ }^{2}$ Department of Early Childhood Education, Faculty of Education and Human Development, Education University of Hong Kong, Hong Kong, HKSAR, China ${ }^{3}$ School of Social Work, Indiana University-Purdue University, Indianapolis, Indiana, USA

${ }^{4}$ California Institute for Behavioral Health Solutions, Sacramento, California, USA 
Acknowledgements We would like to thank the service users who have provided the research team with feedback on the early version of the questionnaire and service users who will be participating in the trial.

Contributors ST and RJG conceived the study. ST, SMCN, WYWY, RJG and SF contributed to the study design and they are the research grant holders (named investigators). ST and WYWY prepared the initial proposal for funding application. SMCN, RJG and SF provided methodological expertise in the study design. ST, SMCN, WYWY and WKIL prepared the first draft of this study protocol. ST, SMCN, WYWY, RJG, SF and WKIL contributed to the rewriting and refinements; all of the authors approved the final manuscript.

Funding The study is funded by the General Research Fund of the Research Grants Council, Hong Kong SAR (Project number: 17609617). The study funder has no role in the study design; collection, management, analysis and interpretation of data; writing of the report; and the decision to submit the report for publication.

Competing interests None declared.

Patient consent for publication Obtained.

Ethics approval The study received ethical approval from the Human Research Ethics Committee of The University of Hong Kong (EA1703078) and is currently recruiting participants.

Provenance and peer review Not commissioned; externally peer reviewed.

Open access This is an open access article distributed in accordance with the Creative Commons Attribution Non Commercial (CC BY-NC 4.0) license, which permits others to distribute, remix, adapt, build upon this work non-commercially, and license their derivative works on different terms, provided the original work is properly cited, appropriate credit is given, any changes made indicated, and the use is non-commercial. See: http://creativecommons.org/licenses/by-nc/4.0/.

\section{REFERENCES}

1. Fukui S, Shimizu Y, Rapp CA. A Cross-cultural study of recovery for people with psychiatric disabilities between U.S. and Japan. Community Ment Health J 2012;48:804-12.

2. Lam LC, Wong CS, Wang MJ, et al. Prevalence, psychosocial correlates and service utilization of depressive and anxiety disorders in Hong Kong: the Hong Kong Mental Morbidity Survey (HKMMS). Soc Psychiatry Psychiatr Epidemiol 2015;50:1379-88.

3. Chang WC, Wong CS, Lam LC, et al. Prevalence, psychosocial and physical health correlates of psychotic disorders in Hong Kong: the Hong Kong Mental Health Morbidity Survey. Schizophrenia Bulletin 2015;41:S135.

4. Whiteford HA, Degenhardt L, Rehm J, et al. Global burden of disease attributable to mental and substance use disorders: findings from the Global Burden of Disease Study 2010. Lancet 2013;382:1575-86.

5. Bloom D, Cafiero E, Jané-Llopis E, et al. The global economic burden of non-communicable diseases. IDEAS Working Paper Series from RePEc 2012.

6. Lee GTH, Yiu MGC. Psychiatric rehabilitation and recovery - situation of Hong Kong. Hong Kong J Ment Health 2013;39:4-12.

7. Anthony WA. Recovery from mental illness: the guiding vision of the mental health service system in the 1990s. Psychosoc Rehabil $J$ 1993;16:11-23.

8. Tse S, Murray G, Chung KF, et al. Exploring the recovery concept in bipolar disorder: a decision tree analysis of psychosocial correlates of recovery stages. Bipolar Disord 2014;16:366-77.

9. Seligman M. The President's address: APA 1998. Annual Report. Am Psychol 1999;54:559-62.

10. Park N, Peterson C, Seligman MEP. Strengths of character and wellbeing. J Soc Clin Psychol 2004;23:603-19.

11. Rapp CA, Chamberlain R. Case management services for the chronically mentally ill. Soc Work 1985;30:417-22.

12. Rapp CA, Goscha RJ. The strengths model: case management with people with psychiatric disabilities. USA: Oxford University Press, 2006.

13. Rapp CA, Goscha RJ. The Strengths Model: a recovery-oriented approach to mental health services. New York: Oxford University Press, 2011.

14. Carlson L, Goscha RJ, Rapp CA. Field mentoring: an important strategy for evidence-based practice implementation. Best Pract Ment Health 2016;12:1-13.

15. Ziguras SJ, Stuart GW. A meta-analysis of the effectiveness of mental health case management over 20 years. Psychiatr Serv 2000;51:1410-21.
16. Mueser KT, Bond GR, Drake RE, et al. Models of community care for severe mental illness: a review of research on case management. Schizophr Bull 1998;24:37-74.

17. Chopra P, Hamilton B, Castle D, et al. Implementation of the strengths model at an area mental health service. Australas Psychiatry 2009;17:202-6.

18. Modrcin M, Rapp CA, Poertner J. The evaluation of case management services with the chronically mentally ill. Eval Program Plann 1988;11:307-14.

19. Macias C, Kinney R, Farley OW, et al. The role of case management within a community support system: partnership with psychosocial rehabilitation. Community Ment Health J 1994;30:323-39.

20. Macias C, Farley OW, Jackson R, et al. Case management in the context of capitation financing: an evaluation of the strengths model. Adm Policy Ment Health 1997;24:535-43.

21. Stanard RP. The effect of training in a strengths model of case management on client outcomes in a community mental health center. Community Ment Health J 1999;35:169-79.

22. Rapp CA, Wintersteen R. The strengths model of case management: results from twelve demonstrations. Psychosoc Rehabil J 1989;13:23-32

23. Ryan CS, Sherman PS, Judd CM. Accounting for case manager effects in the evaluation of mental health services. $J$ Consult Clin Psychol 1994;62:965-74.

24. Kisthardt W. An empowerment agenda for case management research: evaluating the strengths model from the consumers perspective. In: Harris M, Bergman $\mathrm{H}$, eds. Chronic mental illness case management for mentally ill patients: theory and practice. England: Harwood Academic Publishers/Gordon, 1993:165-81.

25. Barry KL, Zeber JE, Blow FC, et al. Effect of strengths model versus assertive community treatment model on participant outcomes and utilization: two-year follow-up. Psychiatr Rehabil J 2003;26:268-77.

26. Tse S, Tsoi EW, Hamilton B, et al. Uses of strength-based interventions for people with serious mental illness: a critical review. Int J Soc Psychiatry 2016;62:281-91.

27. Blow FC, Ullman E, Barry KL, et al. Effectiveness of specialized treatment programs for veterans with serious and persistent mental illness: a three-year follow-up. Am J Orthopsychiatry 2000;70:389-400.

28. Björkman T, Hansson L, Sandlund M. Outcome of case management based on the strengths model compared to standard care. A randomised controlled trial. Soc Psychiatry Psychiatr Epidemiol 2002;37:147-52.

29. Ellis G, King R. Recovery focused interventions: perceptions of mental health consumers and their case managers. AeJAMH 2003;2:67-76.

30. Green CA, Janoff SL, Yarborough BJ, et al. The recovery group project: development of an intervention led jointly by peer and professional counselors. Psychiatr Serv 2013;64:1211-7.

31. Mireau R, Inch R. Brief solution-focused counseling: a practical effective strategy for dealing with wait lists in community-based mental health services. Soc Work 2009;54:63-70.

32. Fukui S, Goscha R, Rapp CA, et al. Strengths model case management fidelity scores and client outcomes. Psychiatr Serv 2012;63:708-10.

33. Bondemark L, Ruf S. Randomized controlled trial: the gold standard or an unobtainable fallacy? Eur J Orthod 2015;37:457-61.

34. Tse S, Divis M, Li YB. Match or Mismatch: Use of the Strengths Model with Chinese Migrants Experiencing Mental Illness: Service User and Practitioner Perspectives. Am J Psychiatr Rehabil 2010;13:171-88.

35. Song L-yu, Shih C-yi. Recovery from partner abuse: the application of the strengths perspective. Int J Soc Welf 2010;19:23-32.

36. Tsoi WS, Tse S, Fukui S, et al. Study protocol for a controlled trial of Strengths Model Case Management in mental health services in Hong Kong. BMJ Open 2015;5:e008303.

37. Muthén LK, Muthén BO. How to use a Monte Carlo study to decide on sample size and determine power. Struct Equ Modeling 2002;9:599-620.

38. Giffort D, Schmook A, Woody C, et al. Recovery assessment scale. Chicago, IL: Illinois Department of Mental Health, 1995.

39. Cook JA, Steigman P, Pickett S, et al. Randomized controlled trial of peer-led recovery education using Building Recovery of Individual Dreams and Goals through Education and Support (BRIDGES). Schizophr Res 2012;136:36-42.

40. Patton M. Qualitative evaluation and research methods. Newbury Park, California: Sage Publications, 1990

41. Muthén B, Asparouhov T. Beyond multilevel regression modeling: multilevel analysis in a general latent variable framework. In: Hox J, Robert J, eds. Handbook of advanced multilevel analysis. New York: Routledge, 2011:15-40. 
42. Hu LT, Bentler PM. Cutoff criteria for fit indexes in covariance structure analysis: conventional criteria versus new alternatives. Struct Equ Modeling 1999;6:1-55.

43. Enders CK. Applied missing data analysis. New York, NY, US: Guilford Press, 2010.

44. Glaser BG. More grounded theory methodology: a reader. Mill Valley, CA: Sociology Press, 1994

45. Tweed A, Charmaz K. Grounded theory methods for mental health practitioners. In: Harper D, Thompson AR, eds. Qualitative research methods in mental health and psychotherapy: a guide for students and practitioners. Chichester, West Sussex: John Wiley \& Sons, 2012:131-46.

46. Charmaz K. Constructing grounded theory: a practical guide through qualitative analysis. London, Thousand Oaks, California: Sage Publications, 2006.

47. Thomas DR. A general inductive approach for analyzing qualitative evaluation data. Am J Eval 2006;27:237-46.

48. Corrigan PW, Giffort D, Rashid F, et al. Recovery as a psychological construct. Community Ment Health J 1999;35:231-9.

49. Corrigan PW, Salzer M, Ralph RO, et al. Examining the factor structure of the recovery assessment scale. Schizophr Bull 2004;30:1035-41.
50. Snyder CR, Sympson SC, Ybasco FC, et al. Development and validation of the State Hope Scale. J Pers Soc Psychol 1996;70:321-35.

51. Elliott G, Kao S, Grant A-M. Mattering: empirical validation of a social-psychological concept. Self Identity 2004;3:339-54.

52. McColl MA, Davies D, Carlson P, et al. The community integration measure: development and preliminary validation. Arch Phys Med Rehabil 2001;82:429-34.

53. Conrad KJ, Yagelka JR, Matters MD, et al. Reliability and validity of a Modified Colorado Symptom Index in a national homeless sample. Ment Health Serv Res 2001;3:141-53.

54. Mak WW, Wu CF. Cognitive insight and causal attribution in the development of self-stigma among individuals with schizophrenia. Psychiatr Serv 2006;57:1800-2.

55. Hatcher RL, Gillaspy JA. Development and validation of a revised short version of the working alliance inventory. Psychotherapy Research 2006;16:12-25.

56. Hsu S, Zhou RDH, Yu CKC. A Hong Kong validation of working alliance inventory - short form - client. Asia Pac J Couns Psychother 2016;7(1-2):69-81.

57. Hsu S, Yu CK-C, Ckc Y. A Hong Kong study of working alliance inventory short form - therapist. Asia Pac J Couns Psychother 2017;8:87-100 